\title{
AMONOIDEOS Y OTROS MACROFÓSILES DEL LECTOESTRATOTIPO DE LA FORMACIÓN LA FRONTERA, TURONIANO INFERIOR - MEDIO (CRETÁCICO SUPERIOR) EN SAN FRANCISCO, CUNDINAMARCA (COLOMBIA)
}

Pedro Patarroyo ${ }^{1}$

DOI: http://dx.doi.org/10.18273/revbol.v38n3-2016003 (c) (1) అ

Forma de citar: Patarroyo, P. 2016. Amonoideos y otros macrofósiles del lectoestratotipo de la Formación La Frontera, Turoniano inferior - medio (Cretácico Superior) en San Francisco, Cundinamarca (Colombia). Boletín de Geología, 38(3): 41-54.

\section{RESUMEN}

Una sucesión de cerca de 109 m de espesor del lectoestratotipo de la Formación La Frontera (Colombia) se describe junto con el contenido fósil de Wrightoceras munieri (Pervinquière, 1907), Vascoceras cf. constrictum (Renz and Álvarez, 1979), V. cf. venezolanum Renz, 1982, Kamerunoceras sp., K. cf. turoniense (d'Orbigny, 1850), Hoplitoides cf. lagiraldae Etayo-Serna, 1979, Codazziceras ospinae (Karsten, 1858), Coilopoceras cf. newelli (Benavides-Cáceres, 1956); bivalvos: Anomia colombiana Villamil, 1996, e inocerámidos; y decápodos y plantas (troncos). Estos ejemplares incluidos dentro de la sucesión de la Formación La Frontera, en su mayoría, representan el Turoniano inferior - medio. La existencia de depósitos del Cenomaniano más alto hacia la base de la Formación La Frontera no se puede demostrar en este trabajo.

Palabras clave: Formación La Frontera, Lectoestratotipo, Amonoideos, Turoniano, San Francisco-Cundinamarca

\section{AMMONITES AND OTHER MACROFOSSILS FROM THE LECTOSTRATOTYPE OF LA FRONTERA FORMATION, LOWER - MIDDLE TURONIAN (UPPER CRETACEOUS) IN SAN FRANCISCO, CUNDINAMARCA (COLOMBIA)}

\begin{abstract}
A succession of about $109 \mathrm{~m}$ thick of the La Frontera Formation lectostratotype (Colombia) is described as well as the fossils Wrightoceras munieri (Pervinquière, 1907), Vascoceras cf. constrictum (Renz and Álvarez, 1979), V. cf. venezolanum Renz, 1982, Kamerunoceras sp., K. cf. turoniense (d'Orbigny, 1850), Hoplitoides cf. lagiraldae Etayo-Serna, 1979, Codazziceras ospinae (Karsten, 1858), Coilopoceras cf. newelli (Benavides-Cáceres, 1956); bivalves: Anomia colombiana Villamil, 1996, and inoceramids; and decapods and plants (woods). These fossils from La Frontera Formation indicate a lower - middle Turonian interval. It could not be confirmed the presence of uppermost Cenomanian deposits in the lower part of the La Frontera Formation.
\end{abstract}

Keywords: La Frontera Formation, Lectostratotype, Ammonites, Turonian, San Francisco-Cundinamarca, Colombia

${ }^{1}$ Departamento de Geociencias. Universidad Nacional de Colombia. Bogotá Cr. 30 N. 45 - 03, pcpatarroyog@unal.edu.co 


\section{INTRODUCCIÓN}

Como parte del proyecto "Estratigrafía de los depósitos del Turoniano del centro de Colombia" (DIB 20101001669 Universidad Nacional de Colombia), la sección de la vereda Tóriba fue reconocida en el curso de Geología de Campo del año 2002 del Departamento de Geociencias de la Universidad Nacional de Colombia, y fue trabajada entre 2003 y 2004, y posteriormente en 2012. Con base en el trabajo de campo y la literatura, fue posible proponer la sección de San Francisco (Cundinamarca) como lectoestratotipo de la Formación La Frontera (Blanco et al., 2004). Esta primera publicación permitió la descripción litoestratigráfica. El presente informe tiene por objeto describir la distribución estratigráfica de los macrofósiles.

La sección de la vereda Tóriba, paralela al río San Miguel, está localizada al NE de San Francisco (Cundinamarca), y a $42 \mathrm{~km}$ al NW de Bogotá (FIGURA 1).

\section{CONSIDERACIONES ESTRATIGRÁFICAS}

Los depósitos de la Formación La Frontera se presentan por encima de las sedimentitas de la Formación Pacho, y por debajo de las de la Formación Conejo (Acosta y Ulloa, 2001a, b; Guerrero, 2002; Blanco et al., 2004). El término Formación Simijaca (Ulloa y Rodríguez, 1991) es aquí considerado inválido, ya que corresponde a los depósitos de la parte inferior y media de la Formación San Rafael, por lo que en algunos casos se interpretó igualmente como la parte inferior y media de la Formación La Frontera. En otros casos su posición estratigráfica genera confusión, ya que fue cartografiada por debajo o por encima de la Formación Hiló (ver mapa de la Plancha 208 Villeta: Acosta y Ulloa 2001a).

El término Pacho fue introducido por Ulloa (1982) para una sucesión grueso granular y bioclástica, presente a los lados del núcleo del Sinclinal de Villa Gómez, al occidente de Pacho (Cundinamarca). Acosta (1993) interpreta una serie de cambios de facies de las formaciones Hiló, Pacho, Arenisca de Chiquinquirá y Simijaca, que generan una gran confusión por el desconocimiento regional del comportamiento de las unidades y sus posiciones estratigráficas relativas. Sobre la base de los trabajos de Acosta y Ulloa (2001a, b) se denota la confusión en la ubicación estratigráfica asignada a la Formación Pacho, ya que la fauna del Albiano, que se cita de acuerdo con Royo y Gómez (1941) en Acosta y Ulloa (2001a, b), en realidad corresponde a la de los depósitos de la infrayacente Formación Hiló. En el mapa geológico de la Plancha 208 de Acosta y Ulloa (2001a) claramente se visualiza que desde el Sinclinal de Villa Gómez hasta Pacho, al oriente, se cartografió toda la sucesión como Formación Pacho, es decir que se incluyen depósitos albianos fino granulares de las formaciones Capotes e Hiló, y por otra parte depósitos cenomanianos grueso granulares y bioclásticos correspondientes a la Formación Pacho. La posición estratigráfica de la Formación Pacho por encima de la Formación Hiló es del Cenomaniano, correlativa con las formaciones Arenisca de Chiquinquirá, Churuvita, Calizas del Salto, Maraca, parte baja de Capacho y para algunos autores la parte alta de la Formación Une.

Por otro lado, para los depósitos suprayacentes a la Formación La Frontera se usa la denominación Formación Conejo, que fue introducida por Renzoni (1981) con localidad tipo en cercanías de los municipios de Oicatá y Chivatá (Boyacá). Se trata de una unidad litoestratigráfica del Macizo de Floresta que para ese sector suprayace a la Formación San Rafael.

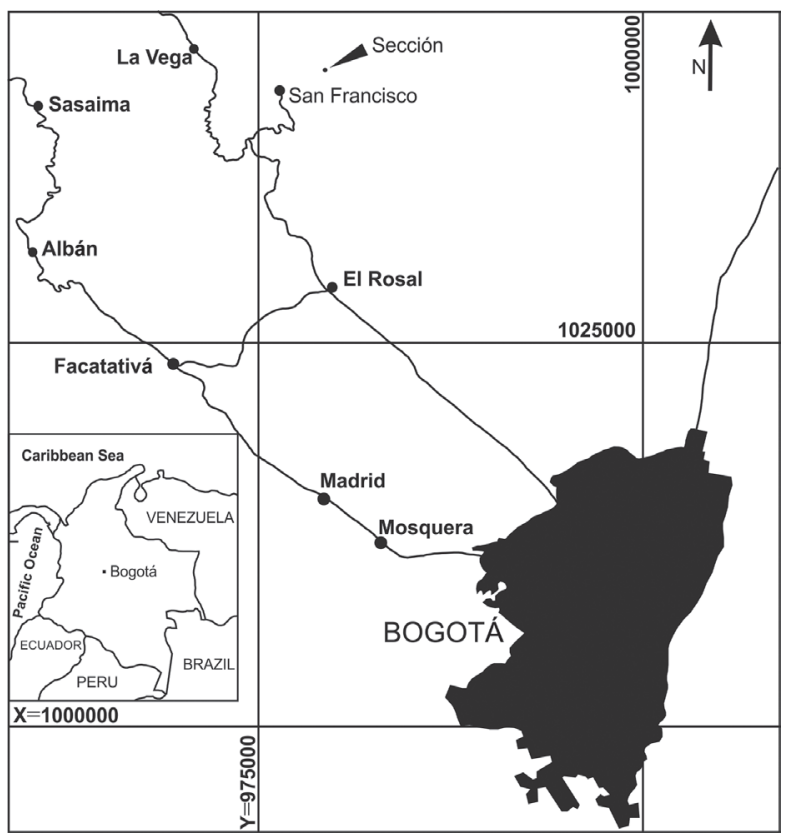

FIGURA 1. Ubicación de la sección de la Formación La Frontera en la vereda Tóriba (San Francisco - Cundinamarca).

\section{FORMACIÓN LA FRONTERA}

Según Blanco et al. (2004) es incorrecto considerar que Hubach (1931a) haya propuesto una sucesión de los afloramientos en Albán (Cundinamarca) como sección tipo de la Formación La Frontera (ver Martínez y Vergara, 1999, p.110). De la revisión bibliográfica se establece que no existe una descripción estratigráfica a lo largo de una sección en Albán. Hay solamente mención en un esquema estratigráfico en Hubach (1931b, pl. 9) 
en relación con el "nivel de La Frontera". Del mismo modo, Hubach (1957, p.102) se refiere al "nivel guía de liditas concrecionarias de La Frontera". Muy a pesar de lo anteriormente expuesto, Martínez y Vergara (1999) indican que la "sección tipo" de la Formación La Frontera en Albán está pobremente expuesta, en donde aflora cerca del 20\% de la sucesión. Martínez y Vergara (1999) se basan en el NACSN (1983) y proponen una sección de referencia principal a partir de la descripción de una sucesión compuesta para la Formación La Frontera, reconociendo que el límite inferior no está bien expuesto.

Idealmente, un neoestratotipo o un lectoestratotipo debe ser propuesto en la localidad tipo, que en este caso corresponde al sector de La Frontera en Albán.

En Albán, el sector de La Frontera, se ubica al noreste del casco urbano, adelante del desvió hacia Guayabal de Síquima. Por la vía del tren se localiza el paradero La Frontera del Ferrocarril de Cundinamarca (Hubach, 1951, p.5), mientras que por la vía pavimentada Albán Villeta, se encontraba la cantera La Frontera, muy cerca de la estación La Frontera, de donde se deduce la cita de Martínez y Vergara (1999). Para este sector, sobre la quebrada La Cochenda, por la vía pavimentada y por el trazado de la línea del tren se encuentran afloramientos aislados de lodolitas físiles, negras, piritosas; biomicritas, concreciones calcáreas, piritosas, bioesparitas y lodolitas silicificadas con amonitas e inocerámidos aplastados del Turoniano. Estratigráficamente abajo aparecen capas gruesas a muy gruesas de cuarzo arenitas bioperturbadas de la Formación Pacho.

En este caso, teniendo en cuenta que no se estableció, ni se describió una sección tipo originalmente, siguiendo los lineamientos del NACSN (1983, en Blanco et al., 2004), NACSN (2005) y de la Guía Estratigráfica Internacional (Salvador, 1994, p.28), Blanco et al. (2004) propusieron un lectoestratotipo para la Formación La Frontera en la vereda Tóriba (San Francisco - Cundinamarca) al norte de Albán, teniendo en cuenta la continuidad y buena exposición que se presenta, los fósiles que se muestrearon y la claridad del límite inferior y superior, muy a pesar que no se encuentra dentro de la localidad tipo, pero si dentro del área tipo.

La sucesión de la Formación La Frontera, sobre el flanco oriental del Anticlinal de San Miguel (San FranciscoCundinamarca, ver Blanco et al., 2004), posee un espesor de 109 m, suprayace arenitas y lodolitas de la Formación Pacho y subyace lodolitas físiles de la localmente denominada Formación Conejo (Blanco et al., 2004). El primer segmento es de $70 \mathrm{~m}$; su parte inferior de $48 \mathrm{~m}$ (niveles I y II de Blanco et al., 2004) está conformada principalmente por intercalaciones de capas delgadas de biomicritas, lodolitas físiles y pequeñas concreciones calcáreas, fosilíferas. Su parte alta de $22 \mathrm{~m}$ (nivel III de Blanco et al., 2004) está caracterizada principalmente por biomicritas, lodolitas calcáreas y grandes concreciones calcáreas, fosilíferas, que pueden contener troncos recristalizados en calcita (FIGURA 2).

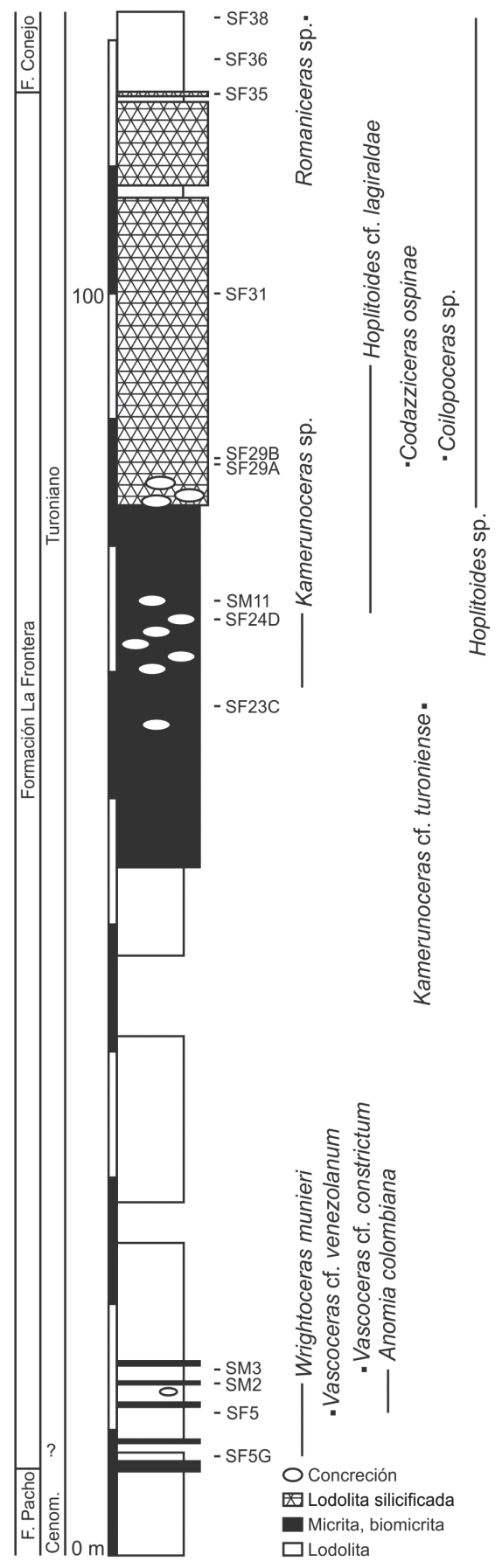

FIGURA 2. Sucesión estratigráfica del lectoestratotipo de la Formación La Frontera (vereda Tóriba, San Francisco Cundinamarca) con la distribución estratigráfica de macrofósiles. 
El segundo segmento tiene $39 \mathrm{~m}$ de espesor (niveles IV y V de Blanco et al., 2004) y está representado por intercalaciones de lodolitas silicificadas, capas delgadas de lodolitas físiles y piríticas, y concreciones calcáreas. La mayoría de los amonoideos, inocerámidos y crustáceos en este segmento se presentan aplastados.

De acuerdo con Etayo-Serna (1979), los depósitos de la Formación La Frontera son del Turoniano, mientras que Villamil (1996, 1998), Villamil and Arango (1998) y Blanco et al. (2004) los consideran del Cenomaniano más alto al Turoniano medio. Para el presente trabajo sólo puede aceptarse un intervalo Turoniano inferiormedio, ya que de acuerdo con la bioestratigrafía para el GSSP de la base del Turoniano (Kennedy et al., 2005) no se cuenta con argumentos de correlación para corroborar la propuesta de Villamil $(1996,1998)$ y Villamil and Arango (1998).

\section{PALEONTOLOGÍA SISTEMÁTICA}

En relación con la taxonomía de las amonitas, se siguen principalmente los lineamientos de Wright et al. (1996) del mismo modo en algunos casos se emplea la nomenclatura abierta de acuerdo con Bengtson (1988), teniendo en cuenta que algunos ejemplares son de talla pequeña. Al igual que lo menciona Patarroyo (2011), no es claro si la talla pequeña de algunos ejemplares colectados obedece a condiciones ecológicas o a fenómenos de mortalidad. Sumado a esto, la recristalización de gran parte de los fósiles presentes no permite determinar, en la mayoría de los casos, si se conserva o no la cámara de habitación.

Las medidas de los ejemplares $D$ (diámetro), $W h$ (altura de la última vuelta), $W w$ (ancho de la última vuelta) y $U$ (diámetro umbilical) se dan en $\mathrm{mm}$, y sus relaciones en porcentajes relativos a $D$.

El repositorio de los individuos aquí descritos se encuentra en las Colecciones Paleontológicas del Departamento de Geociencias de la Universidad Nacional de Colombia - Bogotá.

Orden Ammonoidea

Suborden Ammonitina

Superfamilia Acanthoceratacea

Familia Pseudotissotiidae

Subfamilia Pseudotissotiinae

Género Wrightoceras

Especie tipo Bauchioceras (Wrightoceras) wallsi (Reyment) 1954

Wrightoceras munieri (Pervinquière, 1907) PLANCHA 1, FOTOS 1-7.
Material: ejemplares SM2 $[D=26,4 ; W h=20,2$ $(0,77 \%) ; W w=7,7(0,29 \%) ; W w / W h=0,38 \% ; U=4,1$ $(0,16 \%)]$ y SF5G $[D=20,9 ; W h=11,6(0,6 \%) ; W w=$ $7,3(0,35 \%) ; W w / W h=0,63 \% ; U=2,9(0,14 \%)]$.

Descripción: concha subinvoluta con ombligo estrecho a muy estrecho $(0,14-0,16 \%)$ de acuerdo con Korn (2010). Sección de la vuelta subtrapezoidal a levemente ovalada, más alta que ancha, zona ventral redondeada, plana a levemente convexa. La ornamentación es muy fina y muestra líneas de crecimiento falciformes, que cruzan el vientre sin interrupción y suavemente proyectadas hacia el peristoma.

Observaciones: el individuo SM 2 es muy parecido a Imlayiceras washbournei Leanza, 1967 (véase e,g, Kennedy et al., 1987, fig. 11), a Hoplitoides inca Benavides-Cáceres, 1956 (p.475-476, pl. 63, fig. 6-11), a $H$. wolthmanni (von Koennen, 1897) en Reyment (1972, fig. 3 y 7 (5)) y a W. munieri en Zaborski (1990a), aunque los ejemplares ilustrados aquí tienen el ombligo un poco más abierto. Kennedy et al. (1987, p.58-61) y Chancellor et al. (1994, p.96-100) indican que $H$. inca de Benavides-Cáceres (1956), Imlayiceras? ralphimlayi de Etayo-Serna (1979) y H. munieri de Renz (1982) serian sinónimos de $W$. munieri.

Para Wright et al. (1996) Imlayiceras (Leanza, 1967), es un sinónimo de Wrightoceras (Reyment, 1954), mientras que para Chancellor et al. (1994) y Barroso-Barcenilla and Goy (2007, p.480) Imlayiceras washbournei podría estar en sinonimia con $W$. munieri.

Hoplitoides munieri Pervinquière (1907 pl. X, fig. 1-2,) muestra moldes internos de individuos con la sección de la vuelta subtrapezoidal, ombligo relativamente cerrado y zona ventral aplanada. Los ejemplares de $W$. munieri ilustrados por Kennedy et al. (1987), pl. 10, fig. 10-11), Chancellor et al. (1994, pl. 28, fig. 1-4, pl. 29, fig. 3-8, pl. 36, fig. 1-2), Villamil (1998, fig, 5Q, como H. munieri), BarrosoBarcenilla and Goy (2007, fig. 10, 3-4) y Kennedy et al. (2008, pl. 2, fig. 7, pl. 7, fig. 3, pl. 9, fig. 1-6) muestran principalmente las sinuosidades de las líneas de crecimiento sobre el flanco, ya sea en la concha o en los moldes internos. "Hoplitoides" cf. munieri en Kummel and Decker (1954, fig. 7A, pl. 33, fig. 1-2) presenta ombligo estrecho, surco ventral, sección de la vuelta comprimida y arqueada. H. wohltmanni en Reyment (1972, fig. 3, 7 (5)) muestra la zona ventral suavemente aplanada, apertura umbilical estrecha y la leve ornamentación sobre el flanco, características que son similares al ejemplar SM 2. 


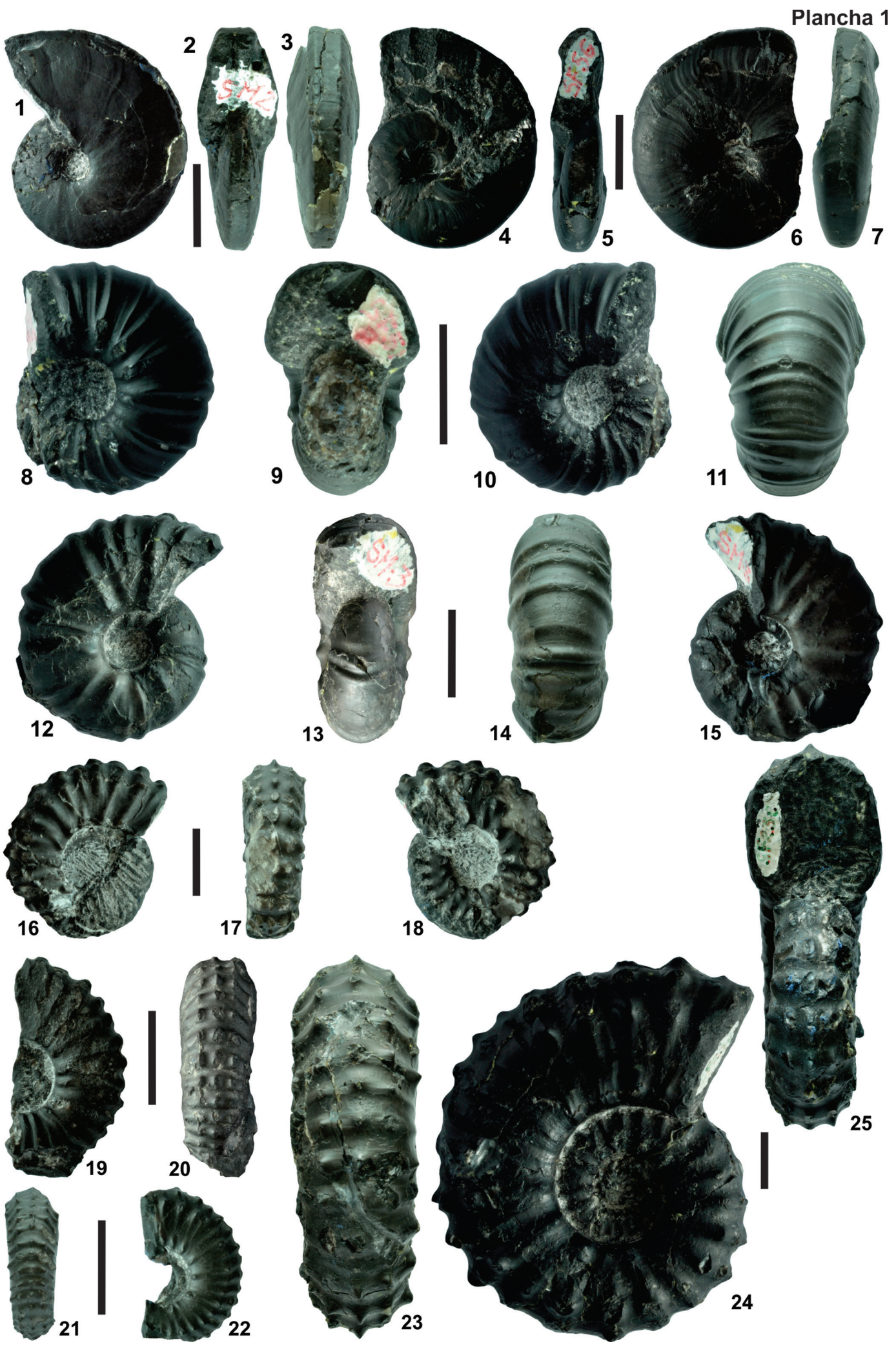

PLANCHA 1. FOTOS 1-7. Wrightoceras munieri (Pervinquière, 1907) (SM2 y SF5G), FOTOS 8-11. Vascoceras cf. venezolanum Renz, 1982 (SF5F), FOTOS 12-15. Vascoceras cf. constrictum (Renz and Álvarez, 1979) (SF5F), FOTOS 16-22. Kamerunoceras sp. (SF24D, SM11-1 y 2), FOTOS 24-25. Kamerunoceras cf. turoniense (d'Orbigny, 1850) (SF23C). Escala 10 mm. 
Edad: para la mayoría de los autores, W. munieri (Pervinquière, 1907) se presenta en el Turoniano inferior (ver Kennedy et al., 1987, p.61; Zaborski, 1990a), Meister and Piuz (2013, fig. 3, p.91) la asocian al bioevento G3 del Turoniano inferior, y BarrosoBarcenilla and Goy (2007, p.481), Kennedy et al. (2008) y Barroso-Barcenilla et al. (2009) la asignan a la parte alta del Turoniano inferior. Para Wright et al. (1996, p.179), Wrightoceras es del Turoniano inferior a medio. Mientras que para Villamil (1996, 1998, p.189), Villamil and Arango (1998, p.133) y Blanco et al. (2004) posee un rango desde el Cenomaniano más alto al Turoniano.

Familia Vascoceratidae

Género Vascoceras

Especie tipo Vascoceras gamai Choffat, 1898

Vascoceras cf. venezolanum Renz, 1982

PLANCHA 1, FOTOS 8-11.

Material: ejemplar SF5F $[D=23,8 ; W h=11,7(0,49 \%)$; $W w=15,2(0,64 \%) ; W w / W h=1,3 \% ; U=6,1(0,26 \%)]$.

Descripción: sección de la vuelta oval deprimida, más ancha que alta. Tubérculos periumbilicales relativamente gruesos sobre las costillas principales. Las costillas principales inician tenuemente en la pared umbilical. Las costillas secundarias aparecen desde la parte baja del flanco. Las costillas en general están arqueadas hacia el peristoma sobre el flanco y continúan sin interrupción a lo largo del vientre pero con una suave curvatura proyectada hacia el peristoma. Las costillas no son muy gruesas, en la parte superior de la última vuelta tienden a estar en parejas y algo espaciadas entre dichas parejas. Hacia el mismo sector se presenta bifurcación de las costillas emparejadas desde los tubérculos periumbilicales. El ombligo es estrecho $(0,27 \%$ subinvoluta) de acuerdo con Korn (2010) y la pared umbilical es redondeada.

Observaciones: Ammonites Toroanus Karsten, 1858 (p. $109-110$, pl. IV, 2) y 1886 (pl. IV, 2) muestra ornamentos similares a nuestro ejemplar, pero la sección de la vuelta de dicho individuo es más alta que ancha. Renz (1982) reconoce diferentes formas de $V$. venezolanum, al igual que Zaborski (1990b, fig. 4E-O). El ejemplar ilustrado aquí es similar al de Renz (1982, pl. 23, 11) y al de Zaborski (1990b, fig. 4E), pero debe contemplarse cuál sería su desarrollo hacia una mayor talla, ya que muy probablemente tales ejemplares se han considerado como individuos de una especie diferente. Así que se recomienda realizar investigaciones ontogenéticas para evaluar esta posibilidad.
Edad: Renz (1982) ubica la presente especie en el Turoniano Inferior. Su asociación con Anomia colombiana (Villamil, 1996) (PLANCHA 1, FOTO 17) refuerza esta posición estratigráfica en la parte baja del Turoniano (ver Villamil, 1996).

\section{Vascoceras cf. constrictum (Renz and Álvarez, 1979)} PLANCHA 1, FOTOS 12-15.

Material: ejemplar SM3 [ $D=24,4 ; W h=11,5(0,47 \%)$; $W w=10,3(0,42 \%) ; W w / W h=0,87 \% ; U=6,3(0,26 \%)]$.

Descripción: concha subinvoluta con ombligo estrecho $(0,25 \%)$ siguiendo a Korn (2010), sección de la vuelta más o menos tan alta como ancha, redondeada. Flanco y pared umbilical redondeados. Las costillas principales inician hacia el margen umbilical, tienden a ser rectas sobre el flanco, continúan y cruzan levemente curvadas la zona ventral hacia el peristoma. Se presenta un engrosamiento en las costillas principales cerca al borde umbilical. Las costillas secundarias inician hacia la parte baja o media del flanco. Puede observarse cierta bifurcación desde los tubérculos periumbilicales. Las costillas no son muy abundantes y se pueden agrupar y mostrar espaciamiento entre las diferentes agrupaciones.

Observaciones: Lewesiceras (?) ulloai (Etayo-Serna 1979, pl. 13, 7) muestra características similares, al igual que Nannovascoceras constrictum Renz and Álvarez (1979) en Renz (1982, pl. 22, 10), pero la ilustración de Etayo-Serna (1979) no permite determinar si los ornamentos periumbilicales son iguales o diferentes al ejemplar aquí ilustrado, menos aún cuando en la descripción no se menciona engrosamientos en las costillas.

Vascoceras birchbyi Cobban and Scott (1972) en Cobban et al. (1989, p.48, fig. 89 A-J), presenta ejemplares de talla pequeña con igual recorrido de las costillas y sus engrosamientos. La diferencia radica en el número de costillas por vuelta, que es mayor en relación con el individuo aquí descrito, por lo que el espaciamiento entre costillas y su distribución no es igual.

Edad: esta especie para Renz and Álvarez (1979, p.978) y Renz (1982) es del Turoniano inferior bajo. Su asociación con Anomia colombiana (Villamil, 1996) (PLANCHA 1, FOTO 16) indica igualmente la parte baja del Turoniano. 


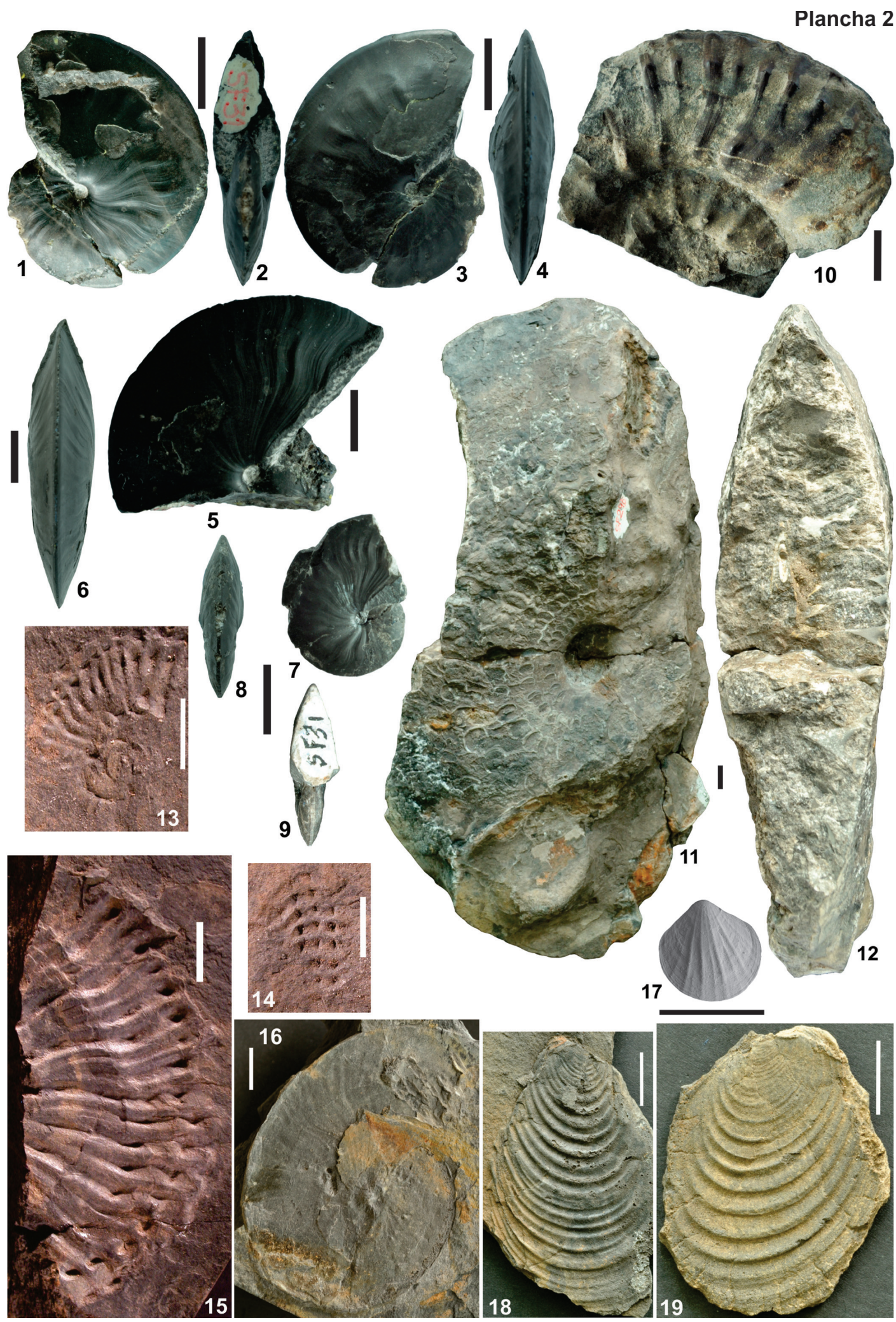

PLANCHA 2. FOTOS 1-9. Hoplitoides cf. lagiraldae Etayo-Serna, 1979 (SM13 y SF31-1 y 2), FOTOS 10. Codazziceras ospinae (Karsten, 1858) (SF29A), FOTOS 11-12. Coilopoceras cf. newelli (Benavides-Cáceres, 1956) (SF29B), FOTOS 1215. Romaniceras sp. (SF38), FOTOS 16. Hoplitoides sp. (SF38), FOTO 17. Anomia colombiana Villamil, 1996, FOTOS 18-19. Inocerámidos (SF36). Escala $10 \mathrm{~mm}$. 
Familia Coilopoceratidae

Género Hoplitoides

Especie tipo Hoplitoides latesellatus (Koenen, 1898)

Hoplitoides cf. lagiraldae Etayo-Serna, 1979

PLANCHA 2, FOTOS 1-9.

Material: ejemplares SM13 $[D=35,8 ; W h=21,6$ $(0,60 \%) ; W w=10(0,28 \%) ; W w / W h=0,46 \% ; U=3,4$ $(0,09 \%)], \mathrm{SF} 31-1[D=33,4 ; W h=20,1(0,60 \%) ; W w=$ 9,1 (0,27\%); Ww/Wh $=0,45 \% ; U=2(0,06 \%)]$ y SF31$2[D=21,7 ; W h=13,3(0,61 \%) ; W w=6(0,28 \%) ; W w /$ $W h=0,45 \% ; U=1,1(0,05 \%)]$.

Descripción: concha involuta, lanceolada (oxicono) y ombligo muy estrecho siguiendo a Korn (2010). La pared umbilical es redondeada y profunda, y las estrías son sinuosas, como ornamentos que nacen en el margen umbilical. Engrosamiento de las costillas hacia la parte alta del flanco.

Observaciones: los engrosamientos periumbilicales de H. lagiraldae Etayo-Serna (1979, pl. 13, fig. 14) no están presentes en los ejemplares aquí descritos, los ornamentos son más similares a $H$. lacabagnae en Etayo-Serna (1979, pl. 13, fig. 9), pero el vientre de los ejemplares aquí tratados es agudo, al igual que los individuos ilustrados por Carvajal y Patarroyo (2007, pl. 1, fig. 3) y Patarroyo (2011, pl. 2, fig. 5-8).

Ejemplares similares a los aquí descritos, han sido identificados como $H$. ingens (Koenen, 1897) (ver Reyment, 1970). H. ingens en Koenen (1898, pl. V, fig. 3, pl. VII, fig. 4-5) posee el borde ventral menos agudo que nuestros ejemplares.

Edad: los ejemplares de $H$. cf. lagiraldae están incluidos en depósitos del Turoniano inferior a medio como en otras sucesiones de Colombia (ver Patarroyo, 2011), y además, debido a que en la sucesión aquí descrita están en asociación faunística con Kamerunoceras, Codazziceras y Coilopoceras. El género Hoplitoides es considerado por Cobban and Kook (1989) con un rango de Turoniano inferior a medio.

\section{Hoplitoides sp. PLANCHA 2, FOTO 16.}

Material: ejemplar SF38

Descripción: ejemplar aplastado y deformado tectónicamente, que muestra ombligo muy estrecho y estrías sinuosas que nacen en el margen umbilical.
Género Coilopoceras

Especie tipo Coilopoceras colleti (Hyatt, 1903)

Coilopoceras cf. newelli (Benavides-Cáceres, 1956) PLANCHA 2, FOTOS 11-12.

Material: ejemplar SF29B [ $D=248 ; W h=125(0,50 \%)$; $W w=68(0,27 \%) ; W w / W h=0,54 \% ; U=22(0,09 \%)]$.

Descripción: fragmento del molde interno de un ejemplar relativamente grande, en el cual se reconocen sectorialmente las líneas de sutura. La concha es involuta con apertura umbilical muy cerrada $(0,09 \%)$ de acuerdo con Korn (2010) y con sección de la vuelta lanceolada. La pared umbilical es redondeada y algo convexa. No se distinguen claramente ornamentos sobre el flanco.

Observaciones: el individuo ilustrado aquí se asemeja al fragmocono de $C$. newelli de Benavides-Cáceres (1956, pl. 61, fig. 4-5), no sólo en las características del flanco y de la sección de la vuelta, además el contorno de la línea de sutura es similar. C. stephani (Renz, 1982) y C. aff. newelli en Renz (1982, pl. 32, fig. 1, pl. 33, fig. 1), C. aff. newelli en Zaborski (1985, fig. 60) y $C$. springeri (Hyatt, 1903) en Kennedy et al. (1989, fig. 29) muestran características similares a las de nuestro ejemplar, sobre los flancos y la apertura umbilical.

Edad: $C$. cf. newelli de la Formación La Frontera se presenta junto con Codazziceras ospinae (Karsten, 1958) y Hoplitoides sp., lo cual estaría indicando el Turoniano inferior a medio (ver Patarroyo, 2011). Estratigráficamente por debajo de este nivel ocurren individuos de Kamerunoceras, por lo que este segmento de la sucesión podría corresponder inclusive, al Turoniano medio. Coilopoceras posee un rango estratigráfico entre Turoniano medio alto a superior bajo de acuerdo con Cobban and Kook (1980).

Familia Acanthoceratidae

Subfamilia Euomphaloceratinae

Género Kamerunoceras

Especie tipo Acanthoceras eschii (Solger, 1904)

Kamerunoceras cf. turoniense (d'Orbigny, 1850) PLANCHA 1, FOTOS 23-25.

Material: ejemplar SF23C $[D=63,8 ; W h=25,2$ $(0,39 \%) ; W w=22(0,34 \%) ; W w / W h=0,87 \% ; U=23,6$ $(0,37 \%)]$.

Descripción: sección de vuelta subcircular, más alta que ancha. Costillas no muy prominentes, las primarias inician en la parte alta de la pared umbilical y las secundarias en la parte media del flanco. Las costillas son rectas a levemente 
curvadas sobre el flanco, suavemente curvas en la zona ventral que cruzan sin interrupción. Enrollamiento subevoluto con apertura umbilical moderada $(0,37 \%)$ siguiendo a Korn (2010). Aparecen siete filas de tubérculos, una en el margen umbilical, en donde son redondeados o elongados pero no en todas las costillas. En el flanco superior los tuberculos pueden ser agudos o redondeados. Los tubérculos ventrolaterales pueden ser agudos y elongados en la dirección del enrollamiento a manera de clavus. Los tuberculos sifonales pueden ser igualmente agudos y elongados en la dirección del enrollamiento. La pared umbical es redonda.

Observaciones: el ejemplar de $K$. eschii (Solger, 1904) de Reyment (1972, fig. 4a-b) es algo similar en costulación a nuestro ejemplar, y muy diferente a la de Reyment (1955, pl. 1, fig. 1, pl. 2, fig. 1), Kennedy and Wright (1979, pl. 1, fig. 4-9), Wright and Kennedy (1981, pl. 14, fig. 4), y Zaborski (1987, fig. 5-6).

K. turoniense en Wright and Kennedy (1981, pl. 14, fig. 2) y Kennedy and Wright (1979, pl. 2, fig. 4-5) muestra igual curvatura de la pared umbilical, el mismo tipo de recorrido de las costillas que nuestro ejemplar, pero la sección de la vuelta es tan ancha como alta a diferencia del individuo de Colombia, que es más alto que ancho. K. turoniense en Barroso-Barcenilla (2004, pl. 3, fig. 2) y Barroso-Barcenilla and Goy (2007, pl. 12D-E, pl. 13A-F) presenta costillas más o menos rectiradiadas y gruesas, sección de vuelta ovalada a redondeada. $K$. turoniense en Zaborski (1993, fig. 6A) ilustra sobre el flanco costillas muy fuertes y menos numerosas. K. puebloense (Cobban and Scott, 1972) muestra en el trabajo de descripción original (pl. 15, fig. 8-9; pl. 37, fig. 1-8; pl. 38, fig. 1) y en Zaborski (1987, fig. 7) ornamentos mucho más fuertes que en nuestro ejemplar. K. salmuriense Courtillier en Collignon (1965, fig. 1659) presenta costillas más espaciadas, levemente rursiradiadas, y enrollamiento similar a nuestro ejemplar.

Edad: Wright and Kennedy (1981) indican que $K$. turoniense se encuentra distribuida desde de la zona de Mammites nodosoides hasta la parte baja de la zona de Collignoniceras woollgari del Turoniano inferior a medio en Inglaterra. Mientras que al lectotipo lo localizan en el Turoniano medio de Francia. Para Kennedy and Wright (1979), K. turoniense indica el Turoniano medio inferior en sectores bien datados con bioestratigrafía. Barroso-Barcenilla (2004) la ubica en la parte baja del Turoniano medio y Amédro and Robaszynski (2008) en el Turoniano medio.

Etayo-Serna (1980) identifica Kamerunoceras n. sp. aff. $K$. turoniense en un intervalo del Turoniano inferior, teniendo en cuenta que se encontró incluida en la zona de asociación de Mammites nodosoidesappelatusFranciscoites suarezi (Etayo-Serna, 1979). Además se halló asociada con Hoplitoides hernanmojicae (EtayoSerna, 1979), Paramammites colombianus (EtayoSerna, 1979), Mammites sp. y Pseudoaspidoceras sp.

\section{Kamerunoceras sp. \\ PLANCHA 1, FOTOS 16-22.}

Material: ejemplares SF24D $[D=24,9 ; W h=9,7$ $(0,39 \%) ; W w=9(0,36 \%) ; W w / W h=0,93 \% ; U=9,3$ $(0,37 \%)]$, SM11-1 $[D=22,4 ; W h=9(\%) ; W w=8(\%)$; $W w / W h=0,89 \% ; U=7,5(0,33 \%)]$ y SM11-2 $[D=16,8$; $W h=6,4(0,38 \%) ; W w=5,4(0,32 \%) ; W w / W h=0,84 \%$; $U=5,2(0,31 \%)]$.

Descripción: el ejemplar SF24D es un molde interno recristalizado en calcita con algo de la concha. Sección de la vuelta levemente más alta que ancha, el margen ventral redondeado, mientras que el flanco tiende a ser recto. Concha subevoluta y apertura umbilical moderada $(0,37 \%)$ de acuerdo con Korn (2010). Costillas primarias de rectiradiadas a levemente prorsiradiadas, que nacen cerca del borde umbilical, sobre las que ocurren tubérculos periumbilicales redondeados a agudos. Las costillas secundarias surgen entre la parte media a alta del flanco. Sobre las costillas aparecen tubérculos elongados en la dirección de enrollamiento (clavus), en la parte alta del flanco, en el borde ventrolateral y sobre el margen sifonal.

Los ejemplares de la muestra SM11-1 y 2 son fragmentos conservados en carbonato de calcio recristalizado, mientras que la SM11-3 es un fragmento piritizado con evidencias de meteorización. Las secciones de las vueltas son redondeadas, levemente más altas que anchas. La concha es subevoluta con apertura umbilical moderada (0.334\% para SM11-1 y 0,309\% para SM112) siguiendo a Korn (2010). Las costillas primarias son rectiradiadas, nacen en el borde umbilical, sobre las que se observan engrosamientos. Las costillas secundarias aparecen desde la parte media del flanco. Sobre las costillas se presentan clavi en la parte alta del flanco, en el borde ventrolateral y sobre el margen sifonal.

Observaciones: la ornamentación de los individuos colombianos tiene mayor semejanza con Kamerunoceras turoniense (d'Orbigny, 1850), pero el tamaño de los ejemplares no permite una determinación contundente.

\author{
Género Codazziceras \\ Especie tipo Lyelliceras scheibei Riedel, 1938 \\ Codazziceras ospinae (Karsten, 1858) \\ PLANCHA 2, FOTO 10.
}


Material: ejemplar SF29A.

Descripción: fragmento de un ejemplar conservado en lodolitas silicificadas, que muestra parte del flanco y de la zona ventrolateral, y que presenta concha evoluta y costillas gruesas, ramificación de las costillas desde los tuberculos umbilicales. El flanco es plano y la pared umbilical redonda. Sólo cuatro filas de tuberculos se pueden observar en el ejemplar descrito. Los tubérculos periumbilicales son los más prominentes con forma elongada a mamilar. Algunos de los tubérculos ventrolaterales son agudos y elongados en dirección del enrrollamiento. Los tubérculos sifonales no son muy notorios.

Observaciones: las costillas principales son gruesas en estado juvenil y subradiales a levemente sinuosas sobre el flanco en estado adulto, tal como se denota en los ejemplares de Wright et al. (1983), Carvajal y Patarroyo (2007, pl. 1, fig. 5) y Patarroyo (2011, pl. 2, fig. 14).

Zaborski (1985, fig. 59; 1990b, fig. 3D) identifica en Nigeria un fragmento de un individuo de C.? ospinae, que presenta igual recorrido de las costillas sobre el flanco plano, tal como ocurre en individuos adultos de Codazziceras, por lo que estos ejemplares deben ser incluidos dentro de este género.

Edad: el individuo de Codazziceras aquí referido, se encuentra junto con individuos de Hoplitoides, lo cual concuerda con la asociación de estos dos géneros en depósitos del Turoniano inferior a medio, como ha sido indicado en Colombia para el Valle Superior del Magdalena (Carvajal y Patarroyo, 2007; Patarroyo, 2011)

\section{Género Romaniceras}

Especie tipo Ammonites deverianus (d'Orbigny, 1841)

\section{Romaniceras sp.}

PLANCHA 2, FOTOS 13-15.

Material: tres ejemplares de la muestra SF38.

Descripción: de la muestra SF38, que corresponde a depósitos de la parte baja de la Formación Conejo, se obtuvieron ejemplares aplastados, sobre lodolita, que muestran la ornamentación del flanco o del margen ventral. Las costillas sobre el flanco son levemente curvadas, las cuales en la parte alta del flanco, a partir de un tubérculo, se proyectan hacia el peristoma produciendo un fuerte quiebre en el recorrido de las costillas. Se reconocen cinco filas de tubérculos, una en el borde umbilical, otra en la parte media del flanco, una tercera en la parte alta del flanco en donde se produce el quiebre de las costillas, otra en el borde ventrolateral y la quinta fila en el margen sifonal. El ombligo podría calificarse como de apertura moderada.

Observaciones: los ejemplares aquí ilustrados se asemejan a Romaniceras (R.) deverianum (d'Orbigny, 1841) en Kennedy et al. (1980, pl. 42, fig. 1-3) y Wrigth and Kennedy (1981, pl. 15, fig. 3), que se asignan a la parte alta del Turoniano medio. En Kennedy and Juignet (2006, p.129, pl. 62, 3 y pl. 63, 1) y Amédro and Robaszynski (2008, p.3) a $R$. deverianum se le ubica en el Turoniano superior.

\section{EDAD DE LA SUCESIÓN}

Para la Formación La Frontera, Villamil (1996, 1998) y Villamil and Arango (1998) indican la ocurrencia de Wrightoceras munieri (Pervinquière, 1907) y fauna de foraminíferos asociados, a partir de lo cual sugieren una posición estratigráfica entre Cenomaniano superior y Turoniano inferior. En tal sentido, Blanco et al. (2004) indican que los depósitos de la base de la Formación La Frontera en la vereda Toriba (San Francisco Cundinamarca) representan el Cenomaniano más alto. Sin embargo, por el momento no se dispone de evidencia significativa para determinar la base del Turoniano.

Así que la fauna de la parte baja del lectoestratotipo de la Formación La Frontera, conformada por $W$. munieri, $V$. cf. constrictum, $V$. cf. venezolanum y Anomia colombiana (PLANCHA 2, FOTO 17), es más característica del Turoniano inferior.

Kamerunoceras sp., K. cf. turoniense, C. ospinae, C. cf. newelli, $H$. cf. lagiraldae se podrían asignar al intervalo Turoniano inferior-medio.

En tal sentido, el rango estratigráfico para la fauna mencionada en este trabajo de la Formación La Frontera, abarca desde el Turoniano inferior al medio.

Hoplitoides sp., Romaniceras sp. e inocerámidos aplastados (PLANCHA 2, FOTOS 13-16, 18-19) de la parte baja de la allí denominada Formación Conejo, podrían indicar el Turoniano medio a superior.

\section{AGRADECIMIENTOS}

Expreso mis agradecimientos a la hoy División de Investigaciones de la Universidad Nacional de Colombia por la financiación del proyecto de investigación "Sedimentitas del Turoniano del centro de Colombia" (DIB-20101001669), a los estudiantes del curso de Geología de Campo de 2002 del Departamento 
de Geociencias (Universidad Nacional de Colombia) por su compañía y asistencia en la sección de la vereda Tóriba (San Francisco - Cundinamarca). Al Servicio Alemán de Intercambio Académico (DAAD, becas A/03/06854, A/06/0736,) y al convenio ALECOLDAAD, MINISTERIO DE EDUCACION NACIONAL DE COLOMBIA, COLCIENCIAS, ICETEX (beca A/09/74196) por la financiación de las estadías académicas en la Universidad de Heidelberg. Al profesor Peter Bengtson de la Universidad de Heidelberg por la colaboración investigativa y todo el apoyo bibliográfico. Igualmente deseo manifestar mis agradecimientos a Diana Carolina Parra y a Jorge Armando Bermúdez por su acompañamiento en la búsqueda y trabajo del sector de La Frontera en Albán (Cundinamarca). Por último, expreso mis más sinceros agradecimientos a los doctores Fernando Barroso-Barcenilla y Horacio Parent por la revisión del manuscrito, ya que con sus valiosos comentarios se enriqueció la versión final.

\section{REFERENCIAS}

Acosta, J. 1993. Cambios laterales en la Formación Hiló. Cuarto Congreso Colombiano de Geología, I, 182195. Medellín.

Acosta, J., y Ulloa, C. 2001a. Geología de la Plancha 208 Villeta. Escala 1:100.000. INGEOMINAS: 83p.

Acosta, J., y Ulloa, C. 2001b. Geología de la Plancha 227 La Mesa. Escala 1:100000. INGEOMINAS: 79p.

Amédro, F., and Robaszynski, F. 2008. Zonation by ammonites and foraminifers of the VraconnianTuronian interval: A comparison of the Boreal and Tethyan domains (NW Europe/ Central Tunisia). Carnets de Géologie / Notebooks on Geology, Brest, (CG2008_L02): 1-5.

Barroso-Barcenilla, F., Goy, A., and Segura, M. 2009. Ammonite zonation of the upper Cenomanian and lower Turonian in the Iberian Trough, Spain. Newsletters on Stratigraphy, 43: 139-164.

Barroso-Barcenilla, F., and Goy, A. 2007. Revision and new data of the ammonite family Pseudotissotiidae in the Iberian Trough, Spain. Geobios, 40 (2): 455-487.

Barroso-Barcenilla, F. 2004. Acanthoceratidae y zonación de ammonites del Cenomaniense superior y del Turoniense inferior en el área de Puentedey, Cuenca Vasco-Cantábrica, España. Coloquios de Paleontología, 54: 83-114.
Benavides-Cáceres, V.E. 1956. Cretaceous system in northern Peru. Bulletin of the American Museum of Natural History, 108: 353-494.

Bengtson, P. 1988. Open nomenclature. Palaeontology, 31 (1): 223-227.

Blanco, J., Medina, P., y Patarroyo, P. 2004. La Formación La Frontera, Sección Vereda Tóriba: Una propuesta para la designación del Lectoestratotipo. Geología Colombiana, 29: 23-40.

Carvajal, J., y Patarroyo, P. 2007. Estratigrafía de la Formación Loma Gorda en la antigua vía Girardot-Nariño (Cundinamarca-Colombia-S. A.). IX Congreso Colombiano de Geología, Bucaramanga Colombia, 13p.

Chancellor, G.R., Kennedy, W.J., and Hancock, J.M. 1994. Turonian ammonite faunas from central Tunisia. Special papers in Paleontology, 50: 1-118.

Cobban, W.A., and Hook, S.C. 1980. The Upper Cretaceous (Turonian) ammonite family Coilopoceratidae Hyatt in the Western Interior of the United States. U. S. Geological Survey, Professional paper, 1192: 1-28.

Cobban, W.A., and Scott, G.R. 1972. Stratigraphy and ammonite fauna of the Graneros Shale and Greenhorn Limestone fauna near Pueblo, Colorado. US Geological Survey, Professional paper, 645: 108p.

Cobban, W.A., Hook, S.C., and Kennedy, W.J. 1989. Upper Cretaceous rocks and ammonite faunas of southwestern New Mexico. New Mexico Bureau of Mines \& Mineral Resources, Mem., 45: 5-137.

Collignon, M. 1965. Atlas des fossiles caracteristiques de Madagascar (Ammonites) (Turonien). Fasciculo XII. Service Geologique Tanarive: 1- 83.

Etayo-Serna, F. 1980. First record in Colombia of Kamerunoceras, a Turonian ammonite genus. Geología Norandina, $1: 27-28$.

Etayo-Serna, F. 1979. Zonation of the Cretaceous of Central Colombia by Ammonites. Publicacion Especial 2, INGEOMINAS, Bogota, 186p.

Guerrero, J. 2002. A Proposal on the Classification of Systems Tracts: Application to the Allostratigraphy and Sequence Stratigraphy of the Cretaceous Colombian 
Basin. Part 2: Barremian to Maastrichtian. Geología Colombiana, 27: 27-49.

Hubach, E. 1931a. Exploración de la región de Apulo - San Antonio - Viota. Boletín de Minas y Petróleos, 4 (25-27): 41-60.

Hubach, E. 1931b. Geología petrolera del Departamento de Norte de Santander. Servicio Geológico Nacional, informe 176. Parte A.

Hubach, E. 1951. Deslizamiento en el FC de Cundinamarca entre Facatativá y Útica. Ministerio de Minas y Petróleos. Informe 723, 7p.

Hubach, E. 1957. Estratigrafía de la Sabana de Bogotá y alrededores. Boletín Geológico, 5 (2): 93 - 112.

Hyatt, A. 1903. Pseudoceratites of Cretaceous. United States Geological Survey Monograph, 44: 1-351.

Karsten, H., 1886. Géologie de l'ancienne Colombie bolivarienne, Venezuela, Nouvelle-Grenade et Ecuador, $60 \mathrm{p}$.

Karsten, H. 1858. Über die geognostischen Verhältnisse des westlischen Columbien, der heutigen Republiken NeuGranada und Equador. Amtl. Ber. über die 32 Versammlg. d. Deutsch. Naturforsch. in Wien, 1856: 80-115.

Kennedy, W.J., and Juignet, P. 2006. Romaniceras deverianum (d'Orbigny, 1841) (Fischer, J.C. Ed.). Révision critique de la Paléontologie Française d'Alcide d'Orbigny. Vol. IV Cephalopodes Crétacés. Backhuys publ., 129p.

Kennedy, W.J., and Wright, C.W. 1979. On Kamerunoceras Reyment, 1954 (Cretaceous Ammonoidea). Journal of Paleontology, 53: 1165-1178.

Kennedy, W.J., Gale, A., Ward, D., and Underwood, Ch. 2008. Early Turonian ammonites from Goulmima, south Morocco. In: Steurbaut, E., Jagt, J. \& Jagt-Yazykova (Ed.), Annie V. Dhondt Memorial Volume. Bulletin de l'Institut royal des Sciences naturelles de Belgique, Sciences de la Terre, 78: 149-177.

Kennedy, W.J., Walaszczyk, I., and Cobban, W.A. 2005. The Global Boundary Stratotype Section and Point for the base of the Turonian Stage of the Cretaceous: Pueblo, Colorado, U.S.A. Episodes, 28 (2): 93-104.

Kennedy, W.J., Cobban, W.A., Hancock, J.M., and Hook, S.C. 1989. Biostratigraphy of the Chispa Summit
Formation at its type locality: a Cenomanian through Turonian reference section for Trans-Pecos Texas. Bulletin of the Geological Institutions of the University of Uppsala. N. S., 15: 39 - 119.

Kennedy, W., Wright, C., and Hancock, J. 1987. Basal Turonian ammonites from west Texas. Palaeontology, 30 (1): $27-74$.

Kennedy, W., Wright, C., and Hancock, J. 1980. The European species of the Cretaceous ammonite Romaniceras with a revision of the genus. Palaeontology, 23 (2): $325-362$.

Koenen, A. von. 1898. Nachtrag zu Ueber Fossilen der unteren Kreide am Ufer des Mungo in Kamerun. Abhandlungen der Königlichen Gesellschaft der Wissenschaften zu Göttingen, MathematischPhysikalische Klasse, Neue Folge, 1 (1): 49-65.

Koenen, A. von. 1897. Ueber Fossilen der unteren Kreide am Ufer des Mungo in Kamerun. Abhandlungen der Königlichen Gesellschaft der Wissenschaften zu Göttingen, Mathematisch-Physikalische Klasse, Neue Folge, 1 (1): 1-48.

Korn, D. 2010. A key for the description of Palaeozoic ammonoids. Fossil Record, 13 (1): 5 - 12.

Kummel, B., and Decker, J. D. 1954. Lower Turonian ammonites from Texas and Mexico. Journal of Paleontology, 28 (3): $310-319$.

Leanza, A. F. 1967. Algunos ammonites nuevos o poco conocidos del Turoniano de Colombia y Venezuela. Acta Geol. Lilloana, IX: $189-228$.

Martínez, I., y Vergara, L. 1999. La sucesión paleoambiental del Cretácico de la región de Tequendama y oeste de la Sabana de Bogotá, Cordillera Oriental Colombiana. Geología Colombiana, 24: 107-147.

Meister, C., and Piuz, A. 2013. Late Cenomanian-Early Turonian ammonites of the southern Tethys margin from Morocco to Oman: Biostratigraphy, paleobiogeography and morphology. Cretaceous Research, 44: 83-103.

NACSN. 2005. North American Stratigraphic Code. North American Commission on Stratigraphic Nomenclature. AAPG Bull., 89 (11): 1547-1591.

NACSN. 1983. North American Stratigraphic Code. North American Commission on Stratigraphic Nomenclature. AAPG Bull., 67 (5): 841-875. 
d'Orbigny, A. 1850. Prodrome de Paléontologie Stratigraphique Universelle des Animaux Mollusques et Rayonnés, 2. Masson, Paris, pp. 1-428.

d'Orbigny, A. 1841. Paléontologie Française; Description des Mollusques et Rayonnés Fossiles, Térrains Crétacées, Céphalopodes, 1. Masson, Paris, pp. 121-430.

Patarroyo, P. 2011. Sucesión de amonitas del Cretácico Superior (Cenomaniano - Coniaciano) de la parte más alta de la Formación Hondita y de la Formación Loma Gorda en la quebrada Bambucá, Aipe - Huila (Colombia, S.A.). Boletín de Geología, 33 (1): 69-92.

Pervinquière, L. 1907. Carte Géologique de la Tunisie. Études de paléontologie tunisienne, 1. Céphalopodes des terrains secondaires. Paris, de Rudeval, 438p.

Renz, O. 1982. Cretaceous Ammonites of Venezuela. Birk Hause Ed. Basilea, 132p.

Renz, O., and Álvarez, F. 1979. Two new ammonite genera from the Lower Turonian of Venezuela. Eclogae Geologie Helvetia, 72 (3): 973-979.

Renzoni, G. 1981. Geología del Cuadrángulo J-12, Tunja. Servicio Geologico Nacional, Inf. 1546, 30 p. (1967). Boletín Geológico, 24 (2): 31-48.

Reyment, R. 1972. Some Lower Turonian amonites from Trinidad and Colombia. Geologiska Föreningens i Stockholm Förhandlingar, 94: 357-368.

Reyment, R. 1970. Note sur le genre Hoplitoides dans l'Atlantique de Sud. Bull. Soc. géol. de Fr., XII (5): 913-915.

Reyment, R. 1955. The Cretaceous Ammonoidea of southhern Nigeria and the southern Cameroons. Geol. Sur. of Nigeria. Bull., 25: 9-107.

Reyment, R.A. 1954. New Turonian (Cretaceous) ammonite genera from Nigeria. Colonial Geology and Mineral Resources, 4 (2): 149-164.

Salvador, A. 1994. International Stratigraphic Guide: A Guide to Stratigraphic Classification, Terminology, and Procedure, Second Edition. The International Union of Geological Sciences and The Geological Society of America, 214p.

Solger, F. 1904. Die Fossilien der Mungokreide in Kamerun und ihre geologische Bedeutung, mit besonderer Berücksichtigung der Ammoniten. En: Beiträge zur Geologie von Kamerun, 2. E. Schweizerbart'sche Verlagsbuchhandlung, Stuttgart, pp. 85-242.

Ulloa, C. 1982. Proyecto Plancha 208-Villeta. Informe de comisión No. 1. Informe preliminar 209. INGEOMINAS. Bogotá.

Ulloa, C. y Rodríguez, E. 1991 Mapa Geológico de Colombia, Plancha 190 Chiquinquirá. INGEOMINAS. Bogotá.

Villamil, T. 1998. Chronology, relative sea level history and a new sequence stratigraphic model for basinal Cretaceous facies of Colombia. In: Paleogeographic evolution and non-glacial eustacy, northern South America, SEPM Special Publication 58, pp. 161-216.

Villamil, T. 1996. Paleobiology of two new species of the bivalve Anomia from Colombia and Venezuela and the importance of the genus in recognition of the base of the Turonian. Cretaceous Research 17: 607-632.

Villamil, T., and Arango, C. 1998. Integrated stratigraphy of latest Cenomanian and early Turonian facies of Colombia. In: Paleogeographic evolution and non-glacial eustacy, northern South America, SEPM Special Publication 58, pp. 129-159.

Wright, C.W., and Kennedy, W.J. 1981. The ammonoidea of the Plenus Marls and the Middle Chalk. Palaeontographical Society Monographs, 134 (560): 1-148.

Wright, C., Callomon, J., and Howarth, J., 1996. Cretaceous Ammonoidea (Kaesler, R. L. Ed.), Treatise on Invert. Paleontology. Part L. Mollusca 4 Revised, 4, Geol. Soc. Am. and Univ. Kansas, 362p.

Wright, C., Chancelor, G., and Kennedy, W., 1983. The affinities of Codazziceras ETAYO-SERNA, 1979 (Cretaceous Ammonoidea). Cretaceous Research, 4 (4): 341-348.

Zaborski, P. 1993. Some new and rare upper cretaceous ammonites from north-eastern Nigeria. Journal of African Earth Sciences, 17 (3): 359-371.

Zaborski, P. 1990a. The Cenomanian and Turonian (mid-Cretaceous) ammonite biostratigraphy of northeastern Nigeria. Bull. Br. Mus. Nat. Hist. (Geology), 46 (1): 1-18. 
Zaborski, P. 1990b. Some upper cretaceous ammonites from southern Nigeria. Journal of African Earth Sciences, 10 (3): 565-581.

Zaborski, P. 1987. Lower Turonian (Cretaceous) ammonites from south-east Nigeria. Bull. Br. Mus. Nat. Hist. (Geology), 41 (2): 31-66.

Zaborski, P. 1985. Upper Cretaceous ammonites from the Calabar region, south-east Nigeria. Bull. Br. Mus. Nat. Hist. (Geology), 39 (1): 1-72.

Trabajo recibido: mayo 13 de 2015

Trabajo aceptado: febrero 19 de 2016

Manuscrito publicado en internet: marzo 1 de 2016 